

\title{
Enhanced thermoelectric performance in spark plasma textured bulk n-type BiTe2.7Se0.3 and p-type Bi0.5Sb1.5Te3
}

Shekhar D. Bhame, Dhanapal Pravarthana, Wilfrid Prellier, and Jacques G. Noudem

Citation: Appl. Phys. Lett. 102, 211901 (2013); doi: 10.1063/1.4807771

View online: http://dx.doi.org/10.1063/1.4807771

View Table of Contents: http://apl.aip.org/resource/1/APPLAB/v102/i21

Published by the AIP Publishing LLC.

\section{Additional information on Appl. Phys. Lett.}

Journal Homepage: http://apl.aip.org/

Journal Information: http://apl.aip.org/about/about_the_journal

Top downloads: http://apl.aip.org/features/most_downloaded

Information for Authors: http://apl.aip.org/authors

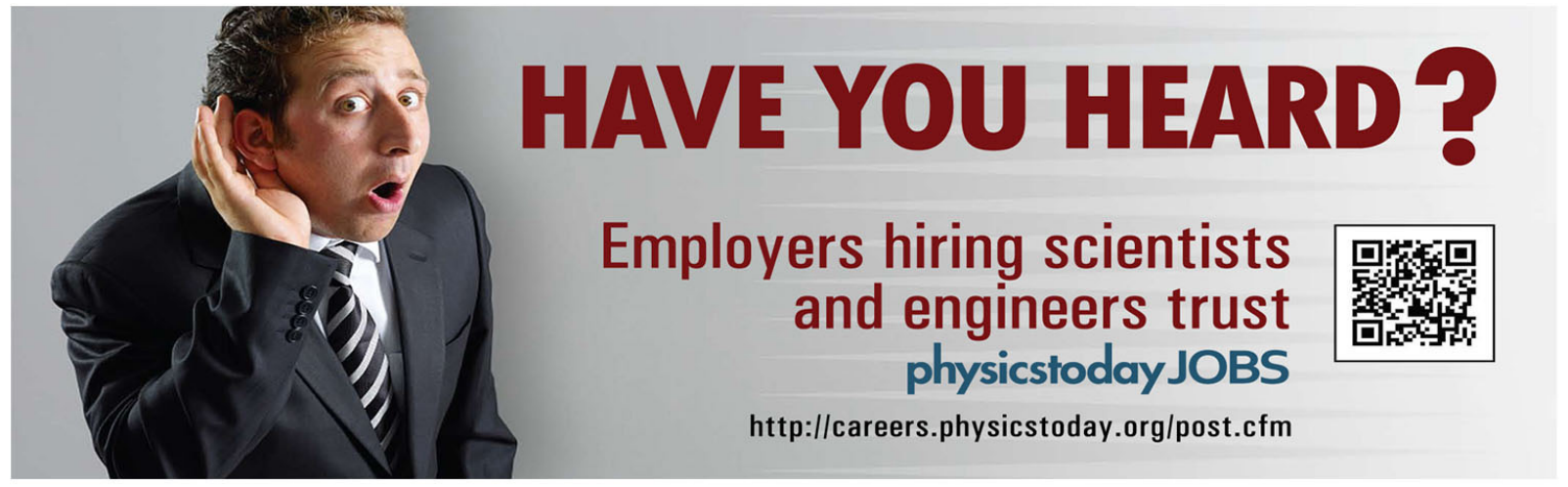




\title{
Enhanced thermoelectric performance in spark plasma textured bulk $n$-type $\mathrm{BiTe}_{2.7} \mathrm{Se}_{0.3}$ and $p$-type $\mathrm{Bi}_{0.5} \mathrm{Sb}_{1.5} \mathrm{Te}_{3}$
}

\author{
Shekhar D. Bhame, ${ }^{1,2, a)}$ Dhanapal Pravarthana, ${ }^{1}$ Wilfrid Prellier, ${ }^{1}$ \\ and Jacques G. Noudem ${ }^{1,2}$ \\ ${ }^{1}$ Laboratoire CRISMAT, CNRS UMR 65086, ENSICAEN, 6 Bld., Marechal Juin, 14050 Caen, France \\ ${ }^{2}$ LUSAC (EA 2607), Université de Caen, Basse Normandie (UCBN), BP 78 , \\ 50130 Cherbourg Octeville, France
}

(Received 1 February 2013; accepted 9 May 2013; published online 28 May 2013)

\begin{abstract}
Bulk $p$ and $n$-type bismuth tellurides were prepared using spark plasma texturization method. The texture development along the uniaxial load in the 001 direction is confirmed from both $\mathrm{x}$-ray diffraction analysis and electron backscattering diffraction measurements. Interestingly, those textured samples outperform the samples prepared by conventional spark plasma sintering (SPS) leading to a reduced thermal conductivity in the ab-plane. The textured samples of $n$-type BiTe $_{2.7} \mathrm{Se}_{0.3}$ and $p$-type $\mathrm{Bi}_{0.5} \mathrm{Sb}_{1.5} \mathrm{Te}_{3}$ showed a $42 \%$ and $33 \%$ enhancement in figure of merit at room temperature, respectively, as compared to their SPS counterparts, opening the route for applications. (C) 2013 AIP Publishing LLC. [http://dx.doi.org/10.1063/1.4807771]
\end{abstract}

The need for renewable and clean sources of energy has been a point of recent concern leading to search for environment friendly materials with low emission of green house gases in order to reduce global warming and to address the continuously increasing energy demands. Thermoelectric (TE) materials are potential candidates for power generation and waste heat recovery in a silent and pollution free manner from heat emitting sources such as automobile exhausts apart from their use in cooling and refrigeration. ${ }^{1,2}$ The performance of TE materials is usually measured by the dimensionless figure of merit ZT defined as, $\mathrm{ZT}=\mathrm{S}^{2} \sigma \mathrm{T} / \kappa$ where $\mathrm{S}$ is the Seebeck coefficient, $\sigma$ is the electrical conductivity, $\mathrm{T}$ is the absolute temperature, and $\kappa$ is the thermal conductivity. ${ }^{1}$ Therefore, materials possessing high electrical conductivity, high Seebeck coefficient, and low thermal conductivity should exhibit relatively high ZT.

Bismuth telluride based thermoelectric materials are till date the best known materials having room temperature $\mathrm{ZT}$ values close to 1 . It is well known that bismuth telluride can be suitably doped with either antimony or selenium to change its thermoelectric properties leading to $p$ and $n$-type behaviors, respectively. Conventionally bismuth telluride is synthesized by zone melting leading to high figure of merit but having poor mechanical properties. A variety of processing routes have been utilized in order to enhance the figure of merit by obtaining a suitable microstructure in polycrystalline bismuth tellurides such as melt spinning, ${ }^{3}$ hot extrusion, ${ }^{4}$ and microwave sintering. ${ }^{5}$ Considerable progress has been done in enhancing the figure of merit of these compounds by nanostructuring ${ }^{6-8}$ and by introducing nano-inclusion. ${ }^{9}$ Spark plasma sintering (SPS) method has been effectively used in recent times for densification of various types of materials. The short sintering times, applicability for wide range of materials from oxides to alloys, industrial scalability has driven the use of this technique in recent times. $^{10,11}$ SPS can significantly change the obtained

\footnotetext{
${ }^{\text {a) }}$ Author to whom correspondence should be addressed. Electronic mail: shekhar.bhame@ensicaen.fr.
}

microstructure of the sintered compact leading to changes in the thermoelectric performance. ${ }^{12}$ For bismuth telluride based materials, the use of spark plasma sintering has been reported by many authors with better thermoelectric properties. ${ }^{12-15}$ Preferential orientation in the direction parallel to the pressure direction has been reported by Euvananont et al. using SPS technique. ${ }^{16}$ Such a texture development is expected to introduce significant anisotropy leading to changes in the electrical and thermal properties. Noudem et al. have recently reported a change in the SPS process to obtain textured 349 thermoelectric oxides. ${ }^{17}$ This process can indeed be applied for inducing texture in several lamellar thermoelectric materials such as bismuth telluride. After repressing, nanosized powders of $n$-type bismuth telluride showed $22 \%$ ZT enhancement. ${ }^{18}$ Here, we present a comparative study on bulk $p$ and $n$-type bismuth telluride using SPS and Spark plasma texturing (SPT), a two step process using SPS and its consequences on the microstructure, texture and thermoelectric performance.

High purity $\left(99.99 \%\right.$ ) $p$-type $\mathrm{Bi}_{0.5} \mathrm{Sb}_{1.5} \mathrm{Te}_{3}$ and $n$-type $\mathrm{BiTe}_{2.7} \mathrm{Se}_{0.3}$ powders were purchased from Metaux Ceramiqus System Engineering (France). SPS samples were prepared at $75 \mathrm{MPa}$ uniaxial pressure at $500{ }^{\circ} \mathrm{C}$ and $450{ }^{\circ} \mathrm{C}$, respectively, for $n$-type and $p$-type powders. For preparing the SPT samples, in first step the $p$ and $n$-type powders were pressed in a $15 \mathrm{~mm}$ graphite die under a low applied pressure of $28 \mathrm{MPa}$ at $200^{\circ} \mathrm{C}$ and $250^{\circ} \mathrm{C}$, respectively, for 5 min thus yielding a compact pellet. In second step, these pellets were kept in a $30 \mathrm{~mm}$ graphite die and the temperature was increased with heating rate of $50{ }^{\circ} \mathrm{C} / \mathrm{min}$ and dwell time of 20 min under the maximum applied pressure of $75 \mathrm{MPa}$. The final sintering temperatures in the SPT samples were $500{ }^{\circ} \mathrm{C}$ and $450^{\circ} \mathrm{C}$, respectively, for $n$-type and $p$-type.

Thermoelectric characterization was carried out using ZEM-3 (ULVAC-RIKO, Inc., Japan) and NETZSCH laser flash and thermal analysis apparatus from room temperature to $250{ }^{\circ} \mathrm{C}$. XRD analysis was carried out using Philips $\mathrm{X}$ 'Pert Pro diffractometer at room temperature. The fracture microstructures were investigated using the high resolution 




FIG. 1. XRD patterns of the n-type (A) and p-type (B) bismuth telluride recorded on the perpendicular surface to the pressure direction for SPS and SPT samples.

Carl Zeiss (Supra 55, Oberkochen, Germany) scanning electron microscope (SEM). The electron backscattering diffraction (EBSD) experiments were performed on fine polished surfaces along parallel and perpendicular direction to the uniaxial load. Automated EBSD scans were performed with a step size of $1 / 10$ th of grain size; the working distance was set to $15 \mathrm{~mm}$ and indexing material data file declared as $\mathrm{Bi}_{2} \mathrm{Te}_{3}$ phase.

Figures 1(a) and 1(b) show the XRD patterns collected on surface perpendicular to the applied pressure along with the simulated pattern for $n$ and $p$-type bismuth tellurides. All peaks could be assigned to rhombohedral structure as previously reported. ${ }^{10,18}$ A comparison of XRD patterns for SPS and SPT samples clearly showed the enhancement in the peak intensity for $(00 l)$ peak (marked with an asterisk symbol) indicating a more lamellar texture formation in SPT samples. Such an enhancement in the intensity of $00 l$ peaks is an indication of preferential orientation of the grains in the pressing direction. Similar enhancement in the $00 l$ peak intensity in the perpendicular direction is reported in literature. ${ }^{19}$ The degree of orientation can be measured in terms of the orientation factor given by

$$
F=\left[\frac{P-P_{0}}{1-P_{0}}\right],
$$

where $P$ and $P_{0}$ represent the ratio of the sum of intensities of the $(00 l)$ reflections to the sum of intensities of all $(h k l)$ and $(00 l)$ reflections for oriented and non-oriented samples, respectively. ${ }^{20}$ Ideally, $\mathrm{F}=1$, for completely oriented and $\mathrm{F}=0$ for nonoriented samples. In the present case, the SPS sintered samples were used as $\mathrm{P}_{0}$, for texture calculation in SPT samples. In the present study, the observed values of $F$ were 0.17 and 0.1 for $n$ and p-type SPT samples, respectively. The higher F shown by n-type is probably due to the higher operational temperatures used for n-type samples. It has been previously shown that operational temperatures can lead to different texture index for $\mathrm{n}$ and $\mathrm{p}$-type bismuth tellurides and has been associated with abnormal grain growth at lower temperatures. ${ }^{21}$
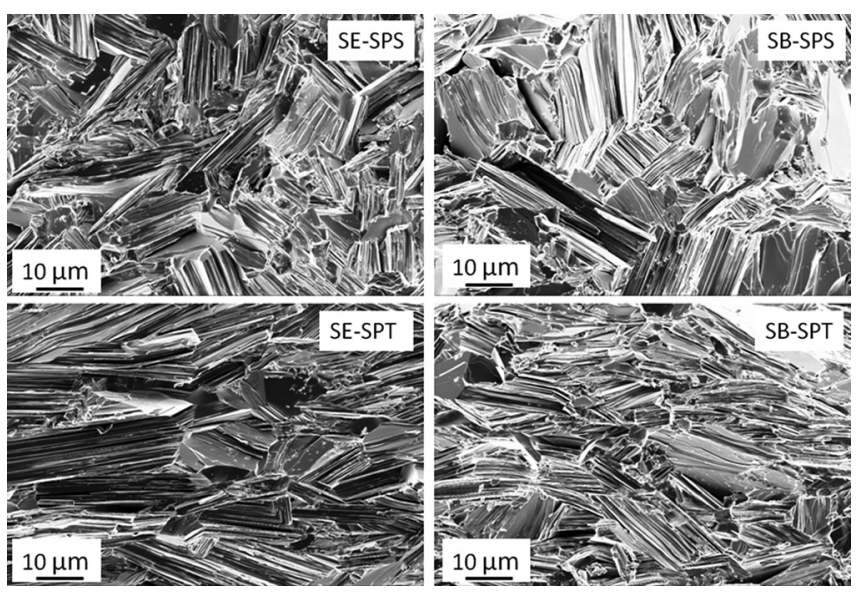

FIG. 2. SEM images of the fracture surfaces of the SPS and SPT samples recorded in the parallel direction to the applied pressure.

The microstructure recorded of the fractured surfaces for both SPS and SPT is shown in Figure 2. For SPS sintering, the grains as well as the lamellar sheets were found to be randomly oriented where as for both SPT samples a clear grain alignment in the flow direction is seen with enhanced lamination to release the strain created by the relatively high applied uniaxial pressure. This clearly indicates that SPT process leads to generation of a enhanced morphological texture in our samples and is consistent with the XRD results. Similar approach has been recently used reorienting the grains in the ab-planes in the case of n-type bismuth telluride. ${ }^{18}$ In order to further confirm the texture development in these samples, EBSD orientation maps of the sample surface parallel to $\mathrm{P}$ (direction of uniaxial load in SPS) illustrate which crystalline direction of each grain aligns with the pressing axis, using a color coded inverse pole figure definition (Figure 3 ). The detailed analysis reveals a significantly large fraction of grains in SPS samples shows random shapes whereas in case of SPT samples, a majority of grains exhibit morphological texture with elongated shape in flow direction (consistent with the SEM results). The uniaxial pressure during the SPS process results in the deformation at high temperature by crystallographic slip in individual grains. Slip should occur on specific crystallographic planes along specific crystallographic directions, and the slip planes are generally planes of high atomic packing density. ${ }^{22}$ The basal plane $\{0001\}$ in bismuth telluride is an easy glide plane. This plane happens to be also the cleavage plane in this material. $^{23}$ The texture computed from the OD for $\{0001\}$ orientation represented in pole figures with orientation densities from minimum to maximum in color coded blue and red boxes, respectively, in multiples of a random distribution (m.r.d) unit. Both the samples prepared by SPS and SPT exhibit crystallographic texture in $\{0001\}$ orientation along the sample transverse direction (direction of uniaxial load in SPS) as the orientation densities with minima to maxima value, see Figure 3. They exhibit annular texture where the main peak in the pole figure is not precisely aligned with the sample transverse direction and the texture is axisymmetric about this peak instead of about the sample transverse direction. The texture direction $\mathrm{T}$ is aligned around $4^{\circ}-5^{\circ}$ for both SPS and SPT samples with the direction of uniaxial load (P) 


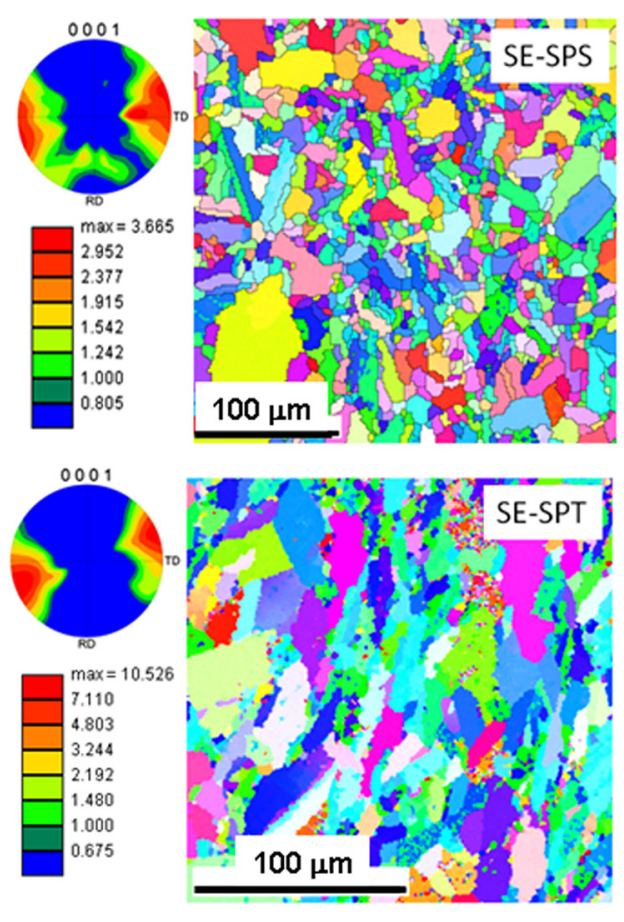

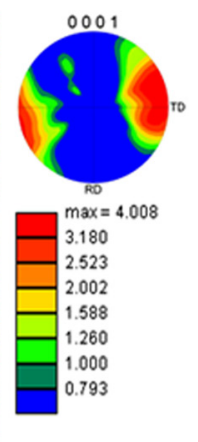
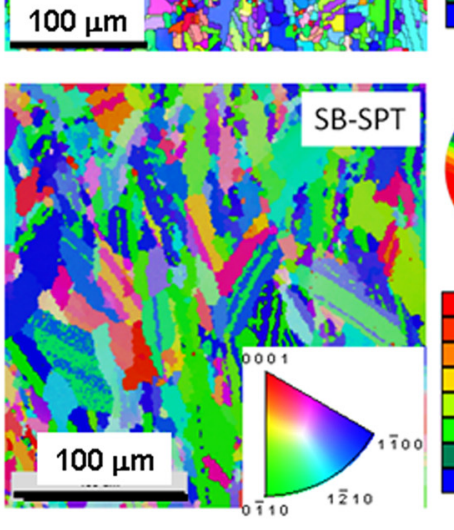

FIG. 3. Color coded Inverse pole figure (IPF) map showing the grains of the SPS and SPT specimens for $\mathrm{N}$ (SE) and P (SB) type bismuth tellurides recorded along the surface perpendicular to uniaxial pressure. The color code corresponding to the crystallographic orientation is given in the stereographic triangle. Pole figure and calculated orientation densities also shown aside. during SPS process. The comparison of orientation densities and the orientation factor (F) for SPT samples clearly shows that for n-type sample we observed higher orientation densities and $\mathrm{F}$ values as compared to the p-type samples. The SPT samples exhibit enhanced texture compared to SPS sample as expected because of plastic deformation due to repressing with high uniaxial load of $75 \mathrm{MPa}$. The formation of preferential orientations of $\{0001\}$ mainly ascribed to significant axial strain in the hot pressing conditions of SPT process crystallographic texture develops due to slip, grain boundary sliding, grain rotations, and dynamic recrystallization. $^{24}$

The electrical resistivity of both the SPS and SPT samples remains almost unchanged but a slight deviation toward lower resistivity for the SPT samples is observed above $150{ }^{\circ} \mathrm{C}$. The room temperature values were found to be almost constant for SPS and SPT samples. While, it is expected that texturization leads to a decrease in the electrical resistivity, in the present case as there are however, more grain boundaries formed in the SPT sample (as compared to SPS sample) exhibiting higher electrical resistivity due to grain boundary scattering which is almost identical to SPS sample. Therefore, a balance between texturization and electrical resistivity occurs leading to an identical behavior between both SPT and SPS.

However a significant change in the Seebeck coefficient is seen between the SPS and the SPT prepared samples. For p-type samples, a 9\% enhancement (from $147 \mu \mathrm{V} / \mathrm{K}$ to $160 \mu \mathrm{V} / \mathrm{K}$ ) in the room temperature Seebeck coefficient was obtained for SPT sample. Similarly in the case of $n$-type SPT sample, the Seebeck coefficient increases from $204 \mu \mathrm{V} / \mathrm{K}$ to $212 \mu \mathrm{V} / \mathrm{K}$, corresponding to $4 \%$ enhancements. According to Mott equation, the Seebeck coefficient is sensitive to band structure near the Fermi level and is not affected by grain orientation. ${ }^{24}$ However for $\mathrm{P}$ type compounds, in the extrinsic conduction region, the Seebeck coefficient can be expressed as



$$
\frac{k_{B}}{e}\left[\delta+2+\ln \frac{2\left(2 \pi m^{*} k_{B} T\right)^{\frac{3}{2}}}{h^{3} n}\right] \text {, }
$$

where $K_{B}$ is Boltzmann constant, a scattering dependent factor, and $h$ is Planck's constant. This has been further simplified for p-type materials as

$$
\frac{k_{B}}{e}[\delta+C-\ln n]
$$

where $\mathrm{C}$ being a constant. This indicates that Seebeck coefficient can be sensitive to the carrier concentration and enhancement can be attributed to the lowering of carrier concentration. We believe that the enhancement in the Seebeck coefficient for SPT samples can also result from change in the carrier concentration in the direction of texture development. ${ }^{10}$ Recently it has been shown that along with carrier concentration and band structure changes at Fermi level, the Seebeck can be affected by the preferential orientation. The $a b$ initio calculations for $\mathrm{p}$ and $\mathrm{n}$ type bismuth telluride performed in parallel and perpendicular direction have shown that the absolute values of Seebeck coefficient were almost identical for $\mathrm{p}$ type but a significant change was observed for $\mathrm{n}$ type bismuth telluride. ${ }^{25}$ Seebeck coefficient measurements on highly textured $\mathrm{Ca}_{3} \mathrm{Co}_{4} \mathrm{O}_{9}$ showed a clear anisotropic behavior. ${ }^{26}$ Yan et al. have also observed a similar enhancement in the Seebeck coefficient after repressing. ${ }^{18}$ Thus, the enhancement in the Seebeck coefficient is attributed to the enhanced texture as observed from XRD and EBSD analysis for SPT samples as compared to SPS prepared samples. The higher Seebeck values obtained for SPT samples lead to an enhancement in the power factor as compared to SPS prepared samples. In the case of p-type bismuth telluride, the maximum power factor changed from $3.190 \mathrm{~W} \mathrm{~m}^{-1} \mathrm{~K}^{-2}$ to $3.857 \mathrm{~W} \mathrm{~m}^{-1} \mathrm{~K}^{-2}$ at room temperature which was $20 \%$ higher than the SPS sample. Similarly for the n-type SPT samples the maximum power factor was obtained at room 


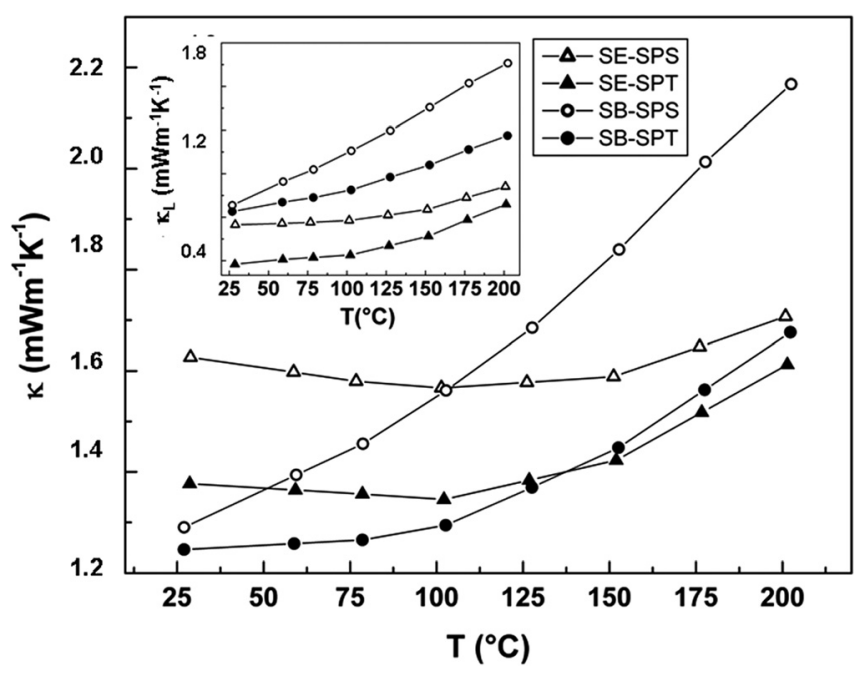

FIG. 4. Temperature dependence of thermal conductivity and lattice thermal conductivity (shown in inset) for SPS and SPT samples.

temperature with a value of $3.72 \mathrm{~W} \mathrm{~m}^{-1} \mathrm{~K}^{-2}$ as compared to $3.47 \mathrm{~W} \mathrm{~m}^{-1} \mathrm{~K}^{-2}$ with $7 \%$ enhancement.

The thermal conductivity measured along the $a b$ direction is shown in Figure 4. The values presented here are experimental for SPS samples; however for the SPT samples, due to lack of sufficiently thick samples we have obtained the $\kappa_{\mathrm{ab}}$ values from $\kappa_{\mathrm{c}}$ measurement. Such a conversion is possible with the results from XRD texture analysis and by using Lotgering method for orientation factor $(F)$ measurements as reported by Shen et al. ${ }^{19}$ A corrected value of thermal conductivity in the $a b$-plane was obtained from the plot of $\kappa_{\mathrm{c}} / \kappa_{\mathrm{ab}}$ against the orientation factor. We have used the same plot used by Shen et al. to calculate $\kappa_{\mathrm{c}} / \kappa_{\mathrm{ab}}$ in present SPT samples. In the present case, for $n$-type and $p$ type SPT samples having $\mathrm{F}$ value 0.17 and 0.1 for $n$ and $p$-type SPT, the ratio of $\kappa_{\mathrm{c}} / \kappa_{\mathrm{ab}}$ was found to be 0.76 and 0.81 , respectively. The obtained ratio was then used to convert the thermal conductivity measured in $c$ direction to thermal conductivity in $a b$ direction. It is well known that both the $n$ and the p-type bismuth tellurides have higher ZT performance in the $a b$-planes than along c-axis. The obtained thermal conductivity values in $a b$-plane of SPT samples were significantly lower than those of SPS samples. For n-type bismuth telluride the thermal conductivity decreased from $1.62 \mathrm{~W} \mathrm{~m}^{-1} \mathrm{~K}^{-1}$ to $1.37 \mathrm{~W} \mathrm{~m}^{-1} \mathrm{~K}^{-1}$ after texturation, where as in the case of $p$-type sample, it changed marginally from $1.29 \mathrm{~W} \mathrm{~m}^{-1} \mathrm{~K}^{-1}$ to $1.24 \mathrm{~W} \mathrm{~m}^{-1} \mathrm{~K}^{-1}$ at room temperature. The total thermal conductivity is a sum of lattice and electronic components, $\kappa_{\mathrm{T}}=\kappa_{\mathrm{L}}+\kappa_{\mathrm{e}}$ and can be separated using Wiedmann-Franz law $\kappa_{\mathrm{e}}=\mathrm{L} \sigma \mathrm{T}$ where $\mathrm{L}$ is Lorentz number and $\mathrm{T}$ is the absolute temperature. From the electrical resistivity measurements, only a slight variation in resistivity for both SPS and SPT is seen. This means that the electronic contribution to total thermal conductivity for both SPS and SPT samples remained almost same in both SPS and SPT samples. Therefore, any reduction in total thermal conductivity can then be attributed to the lattice part of thermal conductivity. Indeed, a significant reduction in the lattice thermal conductivity is seen after texture development in SPT samples as compared to the SPS samples and can be

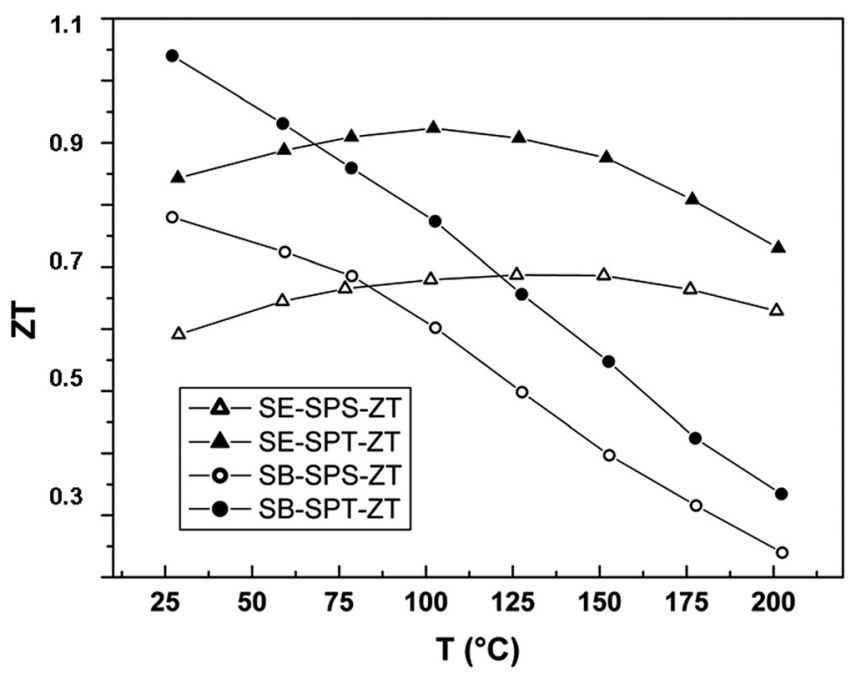

FIG. 5. Figure of merit ZT, for SPS and SPT samples in the case of N and P type bismuth tellurides.

seen in the inset of Figure 4. Therefore, it is very clear that texturization leads to an enhanced scattering at the grain boundary thereby lowering the $\mathrm{K}_{\mathrm{ph}}$. In fact, similar changes in lattice part of thermal conductivity have been observed on preferential grain orientation for bismuth telluride and $\mathrm{Ca}_{3} \mathrm{Co}_{4} \mathrm{O}_{9}$ compounds. ${ }^{18,26}$

Significant enhancement is observed in ZT as shown in Figure 5 for both $n$ and $p$-type after texturization. In the case of $n$-type, the ZT value enhanced from 0.59 for SPS sample to 0.84 for SPT sample at room temperature showing almost $42 \%$ enhancement. A maximum enhancement of ZT to 0.92 which is around $55 \%$ is observed at $100{ }^{\circ} \mathrm{C}$. The p-type SPT sample showed a maximum ZT of 1.04 at room temperature as compared to 0.78 for SPS sample showing $33 \%$ enhancement. The textured samples SPT outperform the conventionally prepared samples by one step SPS process. The grain alignment or preferential orientation in the direction perpendicular the applied pressure leads to texture development in the 001 direction and a greater change in the thermal conductivity consequently enhancing the figure of merit.

In conclusion, we demonstrated the synthesis of textured bulk $p$-type $\mathrm{Bi}_{0.5} \mathrm{Sb}_{1.5} \mathrm{Te}_{3}$ and $n$-type $\mathrm{BiTe}_{2.7} \mathrm{Se}_{0.3}$ using a two steps method in the form of spark plasma texturing. A clear texture development in the 0001 direction parallel to the pressing direction was confirmed from EBSD analysis. As a result of texturation, a significant enhancement in the Seebeck coefficient and a decrease in the thermal conductivity for SPT samples enhanced the thermoelectric figure of merit nearly $33 \%$ for $p$-type and $42 \%$ for $n$-type, respectively, at room temperature, outperforming their SPS counterparts.

D.P. received an Erasmus Mundus $\mathrm{PhD}$ fellowship within the IDS FunMat program supported by the European Commission. Partial support of the French Agence Nationale de la Recherche (ANR), through the program Investissements d'Avenir (ANR-10-LABX-09-01), LabEx $\mathrm{EMC}^{3}$ is also acknowledged.

${ }^{1}$ D. M. Rowe, Renewable Energy 16, 1251 (1999).

${ }^{2}$ L. E. Bell, Science 321, 1457 (2008). 
${ }^{3}$ W. Xie, X. Tang, Y. Yan, Q. Zhang, and T. M. Tritt, Appl. Phys. Lett. 94, 102111 (2009).

${ }^{4}$ C. Andre, D. Vasilevskiy, S. Turenne and R. A. Masut, J. Elec. Mater. 38, 1061 (2009).

${ }^{5}$ G. Delaizir, G. B. Granger, J. Monnier, R. Grodzki, O. Kim-Hak, P.-D. Szkutnik, M. Soulier, S. Saunier, D. Goeuriot, O. Rouleau, J. Simon, C. Godart, and C. Navone, Mater. Res. Bull. 47, 1954 (2012).

${ }^{6}$ Y. Q. Cao, X. B. Zhao, T. J. Zhu, X. B. Zhang, and J. P. Tu, Appl. Phys. Lett. 92, 143106 (2008).

${ }^{7}$ W. Xie, J. He, H. J. Kang, X. Tang, S. Zhu, M. Laver, S. Wang, J. R. Copley, C. M. Brown, Q. Zhang, and T. M. Tritt, Nano Lett. 10, 3283 (2010).

${ }^{8}$ D. H. Kim, C. Kim, D. W. Ha, and H. Kim, J. Alloys Compd. 509, 5211 (2011).

${ }^{9}$ S. Fan, J. Zhao, J. Guo, Q. Yan, J. Ma, and H. H. Hng, Appl. Phys. Lett. 96, 182104 (2010).

${ }^{10}$ G. Molent, M. Thomas, J. Galy, and A. Couret, Adv. Eng. Mater. 9, 667 (2007).

${ }^{11}$ M. Tokita, Am. Ceram. Soc. Bull. 85, 32 (2006).

${ }^{12}$ N. Keawprak, Z. M. Sun, H. Hashimoto, and M. W. Barosum, J. Alloys Compd. 397, 236 (2005).

${ }^{13}$ J. Jiang, L. Chen, S. Bai, and Q. Yao, J. Alloys Compd. 390, 208 (2005).
${ }^{14}$ W. F-Quing, C. Hui, W. Zhong, C. Yan, C. Ying, Z. Lei, and J. Xu-yu, Trans. Nonferrous Met. Soc. China 17, S1010 (2007).

${ }^{15}$ C. H. Kuo, C. S. Hwang, M. S. Jeng, W. S. Su, Y. W. Chou, and J. R. Ku, J. Alloys Compd. 496, 687 (2010).

${ }^{16}$ C. Euvananont, N. Jantaping, and C. Thanachayanont, Curr. Appl. Phys. 11, S246 (2011).

${ }^{17}$ J. G. Noudem, D. Kenfaui, D. Chateigner, and M. Gomina, Scr. Mater. 66, 258 (2012).

${ }^{18}$ X. Yan, B. Poudel, Y. Ma, W. S. Liu, G. Joshi, H. Wang, Y. Lan, D. Wang, G. Chen, and Z. F. Ren, Nano Lett. 10, 3373 (2010).

${ }^{19}$ J. J. Shen, L. P. Hu, T. J. Zhu, and X. B. Zhao, Appl. Phys. Lett. 99, 124102 (2011).

${ }^{20}$ F. K. Lotgering, J. Inorg. Nucl. Chem. 9, 113 (1959).

${ }^{21}$ T. Hayashi, M. Sekine, J. Suzuki, Y. Horio, and H. Takizawa, Mater. Trans. 48, 2724 (2007).

${ }^{22}$ T. H. Courtney, Mechanical Behaviour of Materials (McGraw-Hill, New York, 1990).

${ }^{23}$ A. Sagara and J. W. Faust, J. Appl. Phys. 38, 2410 (1967).

${ }^{24}$ X. A. Fan, J. Y. Yang, W. Zhua, S. Q. Bao, X. K. Duan, C. J. Xiao, and K. Li, J. Alloys Compd. 461, 9 (2008).

${ }^{25}$ B.-L. Huang and M. Kavinay, Phys. Rev. B 77, 125209 (2008).

${ }^{26}$ D. Kenfaui, B. Lenoir, D. Chateignar, B. Ouladdiaf, M. Gomina, and J. G. Noudem, J. Eur. Ceram. Soc. 32, 2405 (2012). 